\title{
Interpreting angular momentum transfer between electromagnetic multipoles using vector spherical harmonics
}

\author{
ROGER GRINTER ${ }^{1}$ AND GARTH A JONES ${ }^{1, *}$ \\ ${ }^{1}$ School of Chemistry, University of East Anglia, Norwich Research Park, Norwich NR4 7TJ, United Kingdom. \\ *Corresponding author: garth.jones@uea.ac.uk
}

Received XX Month XXXX; revised XX Month, XXXX; accepted XX Month XXXX; posted XX Month XXXX (Doc. ID XXXXX); published XX Month XXXX

The transfer of angular momentum between a quadrupole emitter and a dipole acceptor is investigated theoretically. Vector spherical harmonics are used to describe the angular part of the field of the mediating photon. Analytical results are presented for predicting angular momentum transfer between the emitter and absorber, within a quantum electrodynamical framework. We interpret the allowability of such a process, which appears to violate conservation of angular momentum, in terms of the breakdown of the isotropy of space at the point of photon absorption (detection). That is, collapse of the wavefunction results in loss of all angular momentum information. This is consistent with Noether's Theorem and demystifies some common misconceptions about the nature of the photon. The results have implications for interpreting the detection of photons from multipole sources and offers insight into limits on information that can be extracted from quantum measurements in photonic systems.

OCIS codes: 270.0270 Quantum optics, 020.0020; Atomic and molecular physics, 020.5580; Quantum electrodynamics, 270.5585 Quantum information and processing, 270.5565 Quantum communications.

http://dx.doi.org/10.1364/OL.99.099999

Conservation of angular momentum is a fundamental law of physics, being deeply connected to the isotropy of space through Noether's Theorem [1, 2]. Nevertheless the transfer of angular momentum between multipolar species of different leading order (i.e. dipole, quadrupole, octupole, etc.) is not well understood, and can lead to misconceptions about the nature of the photon. The transfer of angular momentum between multipolar centers, via photon exchange, has been considered before $[3,4]$, however not within the context of a spherical description of the fields of the mediating photon. It is advantageous to think about these processes within a spherical context because this forms a natural setting for studying angular momenta. In this work we consider the transfer of angular momentum between two transitions of specific multipole order, within a Coulomb gauge quantum electrodynamical framework, by employing vector spherical harmonics to describe the fields of the photon. We link electrodynamical couplings to projections (i.e. Hermitian scalar products) of the out-going fields of the emitter onto the in-coming fields of the absorber.

The physical process of interest is most easily investigated through an illustrative example. We consider the case of an electronic excited state possessing a downward transition that is electric dipole forbidden, but electric quadrupole allowed. A legitimate question to ask is: To what extent is the nature of the downward transition 'imprinted' onto the emitted photon? The transition energy is defined by the photon's wavevector, but what information about the quadrupole transition moment of the emitter can be extracted from the photon? A quadrupole transition gives rise a change of two units of angular momentum within the electronic system. For example, such a transition could correspond change in the electronic state of an atom from ${ }^{1} \mathrm{D}$ to ${ }^{1} \mathrm{~S}$. One may imagine that if the total angular momentum of the system is to be conserved, the photon could only be absorbed by a similar quadrupole transition that corresponds to an increase in two units of angular momentum. The implication of this is that any dipole transition, whether resonant with the transition energy of the emitter or not, should be transparent to the photon. It is interesting to note that some well-respected quantum mechanics textbooks support this idea through the concept of a "quadrupole photon" [5], and "higher multipole photon" [6], implying that photons originating from transitions of different multipole order are themselves different. This picture is fundamentally incorrect and can lead to considerable confusion. In this work, we show that a photon emitted from a quadrupole source can be absorbed by a dipole transition. This is completely generalizable to multipole transitions of any order. It is important to emphasize that there are a number of papers that acknowledge the existence of finite coupling between species of different leading order in their multipoles [7 - 10]. In this work we show that in analyzing this process in terms of spherical descriptions of the mediating photon, deep insight into the significance of angular momentum conservation within the context of isotropy of space can be gained. Further, there are a number of open questions and active fields of research connected to this topic, including recent investigations that were focused on understanding angular momentum transfer between spin and orbital components [11 - 13], as well as between light and 
matter [14 - 16]. This study aids understanding of the fundamental limitations of encoding information onto photons and may be of interest to those researching structured light $[17,18]$.

Within the framework of quantum electrodynamics (QED), it is has been established experimentally that coupling between a dipole (E1) and quadrupole (E2) can be non-zero [19]. This means that a photon emitted from a downward quadrupole transition can subsequently excite a dipolar transition. Or phrased in another way, photons emerging from quadrupole sources can be registered by dipole detectors. The clear implication of this is that two units of angular momentum are lost from the emitter (E), while only one unit of angular momentum is taken up by the absorber (A), implying that total angular momentum for the electronic system is not conserved for this process.

Photon exchange between an emitter and an acceptor can be understood within the context of the quantum electrodynamical coupling mechanism. The interaction Hamiltonian can be written explicitly for dipole-dipole, quadrupole-quadrupole and dipolequadrupole interactions respectively as $[20,21]$,

$$
\begin{aligned}
& \hat{H}_{\mathrm{int}}^{E 1-E 1}=-\varepsilon_{0}^{-1} \hat{\mu}_{i}(E) d_{i}^{\perp}\left(\vec{R}_{E}\right)-\varepsilon_{0}^{-1} \hat{\mu}_{i}(A) d_{i}^{\perp}\left(\vec{R}_{A}\right) \\
& \hat{H}_{\mathrm{int}}^{E 2-E 2}=-\varepsilon_{0}^{-1} \hat{\Theta}_{i j}(E) \nabla_{i} d_{j}^{\perp}\left(\vec{R}_{E}\right)-\varepsilon_{0}^{-1} \hat{\Theta}_{i j}(A) \nabla_{i} d_{j}^{\perp}\left(\vec{R}_{A}\right) \\
& \hat{H}_{\mathrm{int}}^{E 2-E 1}=-\varepsilon_{0}^{-1} \hat{\Theta}_{i j}(E) \nabla_{i} d_{j}^{\perp}\left(\vec{R}_{E}\right)-\varepsilon_{0}^{-1} \hat{\mu}_{i}(A) d_{j}^{\perp}\left(\vec{R}_{A}\right) .
\end{aligned}
$$

For each case, the first term accounts for photon emission from the source, and the second photon absorption at the detector. $\hat{\mu}_{i}$ is the transition dipole operator, $\hat{\Theta}_{i j}$ is the transition quadrupole operator and $d_{i}^{\perp}$ is the transverse field displacement operator. The matrix element for the electronic coupling can then be calculated explicitly as,

$$
M_{f i}=\sum_{r} \frac{\left\langle f\left|H_{\mathrm{int}}\right| r\right\rangle\left\langle r\left|H_{\mathrm{int}}\right| i\right\rangle}{\left(E_{i}-E_{r}\right)} .
$$

where the summation is over virtual states.

Alternatively, the process can be understood by calculating the energy of a particular multipole in the field of another multipole source. The field, E, generated by a downward multipole transition characterized, by angular momentum quantum numbers $J$ and $M$ is

$$
\begin{aligned}
E=A_{J} e^{i M \varphi} \frac{1}{\sqrt{2 J+1}} & \left\{\sqrt{J+1} h_{J-1}^{(1)}(k r) \mathbf{Y}_{J, J-1, M}(\vartheta, \varphi)\right. \\
& \left.-\sqrt{J} h_{J+1}^{(1)}(k r) \mathbf{Y}_{J, J+1, M}(\vartheta, \varphi)\right\}
\end{aligned}
$$

Where $h_{J+1 / J-1}^{(1)}$ are Hankel functions of the first kind, $A_{J}$ is the amplitude factor that depends on the transition multipole moment and $\mathbf{Y}_{J, J \pm 1, M}$ are vector spherical harmonics (VSH), which as with their scalar counterparts, are eigenfunctions of the angular momentum operator and its z-component. [22, 23] It is important to note that fields are classical objects generated by quantum mechanical operators. The difference between the two treatments, Eqs. (2) and (3), lies in the description of the electromagnetic radiation. Within the context of multipole QED, the interaction Hamiltonian is typically written in terms of a plane wave (PW) expansion, while the latter case exploits angular momentum theory to express multipole transition within a spherical wave (SW) framework.
A spherical wave description of EM radiation is often more advantageous than a plane wave description of light, for example in the case of highly symmetric and isotropic systems. In particular there is much value in considering general aspects of angular momenta associated with photons from the point of view of a spherical description of their fields.

Within the context of QED, field operators replace classical fields. The electric field operator is usually written in terms of a PW description of photons as

$$
\begin{aligned}
\hat{E}(\vec{r})=i \sum_{\vec{k}, \lambda}\left(\frac{\hbar c k}{2 \varepsilon_{0} V}\right)^{1 / 2} & \left\{e^{(\lambda)}(\vec{k}) a^{(\lambda)}(\vec{k}) e^{i \vec{k} \cdot \vec{r}}\right. \\
& \left.-e^{*(\lambda)}(\vec{k}) a^{\dagger(\lambda)}(\vec{k}) e^{-i \vec{k} \cdot \vec{r}}\right\}
\end{aligned}
$$

where creation and annihilation operators, $a^{\dagger(\lambda)}$ and $a^{(\lambda)}$ make and destroy a quantaum of light with wavevector $\vec{k}$ and polarization $\lambda$, respectively. In the case of plane polarized light travelling in the zdirection, application of $a^{\dagger \dagger(x)}$ would create a photon of light polarized along the $\mathrm{x}$-axis. To investigate multipolar radiation, one must expand the phase factors, $e^{ \pm i \vec{k} \cdot \vec{r}}$. This can be achieved using a power series based on,

$$
e^{i \vec{k} \cdot \vec{r}}=\sum_{l} \frac{(i \vec{k} \cdot \vec{r})^{l}}{l !}=1+i(\vec{k} \cdot \vec{r})-\frac{(\vec{k} \cdot \vec{r})^{2}}{2}-\frac{i(\vec{k} \cdot \vec{r})^{3}}{6}+\cdots
$$

Specific terms in the series can then be associated with a particular contribution to the multipole. The first term on the right-hand side is associated with the electric dipole, the second term with the electric quadrupole and the magnetic dipole term, and so on. [19] One can immediately see a limitation of the plane wave description of light, namely that different multipole terms are condensed into a single term of the power series expansion.

In the case of a spherical wave, the multipole terms are defined explicitly in terms of the quantum numbers, $J, l$ and $M$. We can reconstruct the electric field operator, Eq. (4), in terms of spherical waves. Following earlier work [24], we can make the transformation;

$$
e_{1 n}(k) e^{ \pm i \vec{k} \cdot \vec{r}} \rightarrow \Omega_{k J M l}^{ \pm n \pi}
$$

with

$$
\begin{array}{r}
\Omega_{k J M l}^{ \pm n \pi}=4 \pi \sum_{J} \sum_{l} \sum_{M} i^{l} \xi_{l}(k r)\left[Y_{l M-n}\left(\vartheta^{\prime}, \phi^{\prime}\right)\right]^{*} \\
\times\langle l 1 M-n n \mid J M\rangle \mathbf{Y}_{J I M}(\vartheta, \phi)
\end{array}
$$

This function represents the sum of the multipole contributions to the radiation, each specified by the quantum numbers $J, l$ and $M$. It explicitly includes the radial functions, $\xi_{l}$, the polarization, $n$ and the parity, $\pi$. The quantum numbers are determined by the selection rules which, for a single transition emitting or absorbing electric multipole radiation, give one value each of $J$ and $M$ plus one value of $l$ $(=)$ in the case of a magnetic multipole transition and two values of $l$ $(J+1$ and $J-1)$ for an electric multipole transition. Using this reformulation, a photon emitted from the origin and propagating along a vector defined by polar angles, $\vartheta^{\prime}$ and $\varphi^{\prime}$ can be defined via the field operator,

$$
\begin{array}{r}
\hat{E}(\vartheta, \varphi, r)=i \sum_{k, n} \sum_{J, M, l}\left(\frac{\hbar c k}{2 \varepsilon_{0} V}\right)^{1 / 2}\left\{\Omega_{k J M l}^{+n \pi}(k) a_{\Omega}(k)\right. \\
\left.-\Omega_{k J M l}^{-n \pi^{*}}(k) a_{\Omega}^{\dagger}(k)\right\}
\end{array}
$$


This field operator is highly discriminating in terms of the contributions associated with $J, M$ and $l$. In the PW description, Eq. (4), it is clear that we need two parameters to define the photon, namely, the wavevector and the polarization. However any information regarding multipole characteristics of the radiation is hidden within the phase factor.

In the SW formulation of the electric field, there are additional terms appearing in Eq. (8) to provide explicit information about the multipole contributions. The creation and annihilation operators, $a_{\Omega}^{\dagger}(\vec{k})$ and $a_{\Omega}(\vec{k})$, can now be chosen to create or destroy an individual photon that is associated with a characteristic type of EM radiation. That is we can explicitly choose a $J$ and an $M$ value. In the PW case of Eq. (4) however, all orders of the multipole expansion are explicitly included. The deconstruction into specific multipolar terms in the SW description is the origin of the additional sum over $J, M$ and $l$ in eq. (8) compared to eq. (4). Equipped with this theory, we can now investigate the transfer of angular momentum between multipole sources.

The SW formulation of EM radiation can explicitly identify different types of radiation emerging from a transition, based upon quantum numbers associated with the source. Specifically, $J$ equates to the total angular momentum where $J=1$ is associated with the dipole, $J=2$ is identified with the quadrupole, etc. If $\mathrm{J}=l$ we have magnetic $2 J$-pole radiation from an electric multipole source. Combining $J=l-1$ and $J=l$ +1 , we get electric $2 J$-pole radiation from an electric multipole source. Eq. (3) can be resolved into its three orthogonal components along unit vectors $\hat{R}, \hat{\Theta}$ and $\hat{\Phi}$, with the spherical polar co-ordinate system naturally decomposing the field into its longitudinal component along $\hat{R}$ and the transverse component along $\hat{\Theta}$ and $\hat{\Phi}$. See references 25 and 26 for details.

Calculations performed in this work focus on a model system of two multipoles; the photon donor (or source) is fixed at the origin and the acceptor (or detector) at an arbitrary distance from the origin, in the direction defined by polar angles $\vartheta^{\prime}$ and $\varphi^{\prime}$. As the photon propagates away from the origin, the VSH describing the photonic fields grows with the radius being equal to the speed of light multiplied by time, ct. In the region between the emitter and the absorber the out-going field generated by the emitter interacts with the in-coming field of the absorber, also expressed by Eq. (3), but with a Hankel function of second kind $[25,26]$. In adherence to the principle of timereversal invariance, when both fields are expressed in a single coordinate system, their interaction, and hence the probability of photon transfer, is proportional to the Hermitian scalar product of the two fields, $E_{J I M}$

$$
E_{J l M}^{*} \cdot E_{J^{\prime} l^{\prime} M^{\prime}}=\Theta_{J I M}^{*} \Theta_{J^{\prime} l^{\prime} M^{\prime}}+\Phi_{J I M}^{*} \Phi_{J^{\prime} l^{\prime} M^{\prime}}+R_{J l M}^{*} R_{J^{\prime} l^{\prime} M^{\prime}}
$$

To compute the total probability of absorption, one must integrate over a finite surface area of the sphere through which the photon may emerge. Hence the probability of photon transfer at the surface of the sphere at $\vartheta^{\prime}$ and $\varphi^{\prime}$ is the scalar product, Eq. (9), integrated over an infinitesimal area on the unit sphere, $d s=\sin \vartheta d \vartheta d \varphi$.

For the case of emission from a dipole source and absorption by a dipole detector, the Hermitian scalar product elements, $E_{1,0, M}^{*} \cdot E_{1,0, M^{\prime}}$, are shown in Table 1 . The results for the dipole-dipole exchange, as expected, show that transfer of a photon occurs only for dipoles of the same value of $M$, i.e. those aligned along the same axis. For any of the allowed transitions, integration over all angular space will produce a total probability of transfer of unity.

Table 1. Hermitian scalar product for a dipole-dipole photon exchange. The rows represent out-going dipole fields and the columns represent in-coming dipole fields.

\begin{tabular}{llll}
\hline & $E_{1,0,+1}$ & $E_{1,0,0}$ & $E_{1,0,-1}$ \\
\hline$E_{1,0,+1}^{*}$ & $-1 / 4 \pi$ & 0 & 0 \\
$E_{1,0,0}^{*}$ & 0 & $-1 / 4 \pi$ & 0 \\
$E_{1,0,-1}^{*}$ & 0 & 0 & $-1 / 4 \pi$ \\
\hline
\end{tabular}

Table 2. The Hermitian scalar products for quadrupole-quadrupole photon exchange. The rows represent out-going quadrupole fields and columns represent in-coming quadrupole fields.

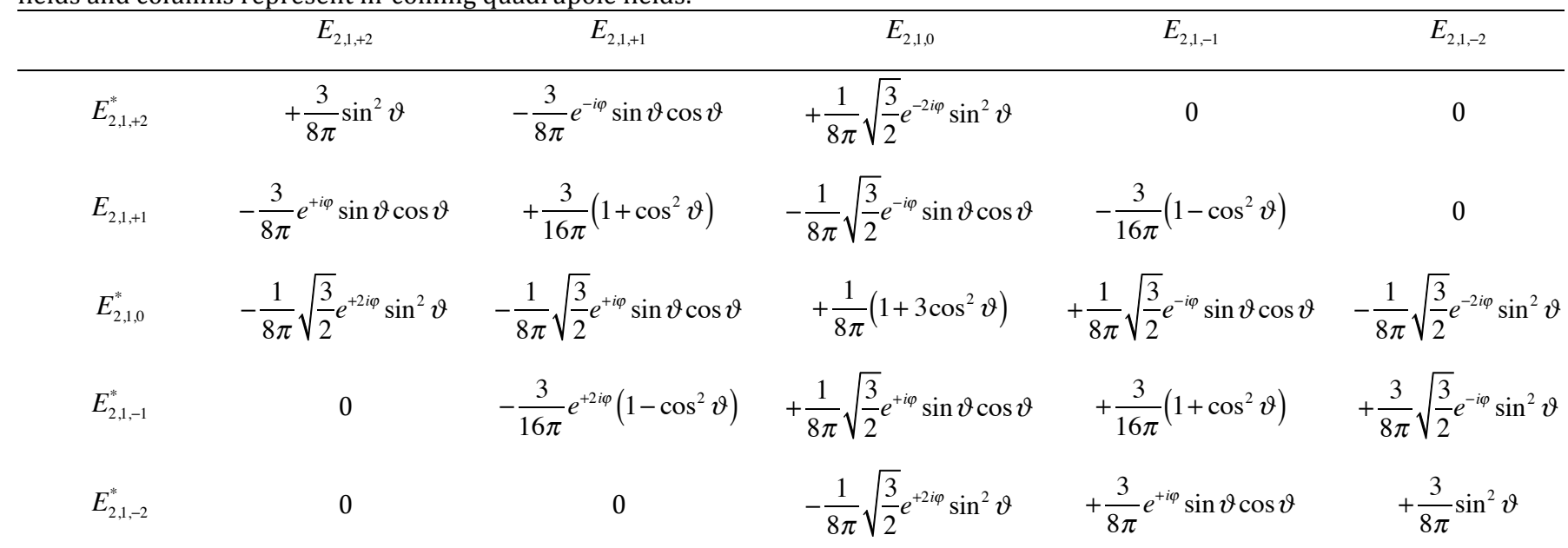

Results of similar calculations for the quadrupole-quadrupole interaction are shown in Table 2, indicating that 19 of the 25 possible transitions are allowed. The six forbidden transitions are those for which the change in $M$ is greater than $|2|$.
The results for the field overlap between the fields of a quadrupole emitter and dipole absorber are displayed in Table 3. The six zero terms correspond to transfer events where $\Delta M \geq|2|$. The existence of the remaining 9 non-zero terms seems to be a counterintuitive result from the perspective of conservation of angular momentum. This implies 
that one unit of angular momentum is transferred from the quadrupole to the dipole, by the mediating photon.

Table 3. Hermitian scalar product of quadrupole-dipole exchange. The rows represent out-going quadrupole fields and the columns incoming dipole fields.

\begin{tabular}{llll}
\hline & $E_{1,0,+1}$ & $E_{1,0,0}$ & $E_{1,0,-1}$ \\
\hline$E_{2,1,+2}^{*}$ & $-\frac{1}{4 \pi} \sqrt{\frac{3}{2}} e^{+i \varphi} \sin \vartheta$ & 0 & 0 \\
$E_{2,1,+1}^{*}$ & $+\frac{1}{4 \pi} \sqrt{\frac{3}{2}} \cos \vartheta$ & $-\frac{\sqrt{3}}{8 \pi} e^{+i \varphi} \sin \vartheta$ & 0 \\
$E_{2,1,0}^{*}$ & $-\frac{1}{8 \pi} e^{-i \varphi} \sin \vartheta$ & $+\frac{1}{2 \sqrt{2} \pi} \cos \vartheta$ & $-\frac{1}{8 \pi} e^{+i \varphi} \sin \vartheta$ \\
$E_{2,1,-1}^{*}$ & 0 & $-\frac{\sqrt{3}}{8 \pi} e^{-i \varphi} \sin \vartheta$ & $+\frac{1}{4 \pi} \sqrt{\frac{3}{2}} \cos \vartheta$ \\
$E_{2,1,-2}^{*}$ & 0 & & $+\frac{1}{4 \pi} \sqrt{\frac{3}{2}} e^{-i \varphi} \sin \vartheta$
\end{tabular}

One may expect that upon emission of a photon via a high order transition (e.g. a quadrupole of an atom), that the photon carries away with it the net loss of angular momentum, and that this photon could only excite the transition of an equivalent multipole. However, we know from previous studies that there is finite coupling between multipoles of different order.

It is interesting to consider what happens when we integrate the Hermitian scalar product results over the surface of the sphere. For all types of interactions considered, namely the dipole-dipole (Table 1), quadrupole-quadrupole (Table 2) and quadrupole-dipole (Table 3), the integration goes to zero, apart from the diagonal elements of the dipole-dipole and quadrupole-quadrupole arrays, each of which integrates to unity. This gives significant insight into the nature of angular momentum transfer between transition multipoles.

In physically interpreting these results it is beneficial to consider the process from the point of view of the geometry of the system. After the photon is emitted by the quadrupole transition but before absorption, we can map the field for all space using, Eq. (3). If the detecting dipole is located on the surface of the sphere at some distance $r=c t$ from the emitter, it 'sees' the photon emerge through a small region of the surface of the spherical shell. That is, in order to calculate the probability of absorption by the dipole at a specific point on the sphere, one needs to integrate over $d s=\sin \vartheta d \vartheta d \varphi$, which gives a finite probability of transfer. However, it is the whole surface of the sphere that contains the complete angular information for the process. Upon photon absorption, all information except for that small area through which the photon emerges is lost. The implication is that once the photon is absorbed, the local isotropy of space around the emitter is broken and angular momentum for the purely electronic process is not conserved. This analysis indicates that the nature of the quadrupole source can only be determined by performing a number of measurements all over the spherical shell surrounding the emitter. The combination of these would allow one to reconstruct the field and hence reveal the nature of the transition.
It is interesting to think about this scenario within the context of quantum measurement and wave-particle duality of the photon.[27] In order to reconstruct the field theoretically, we can use the field operator written in VSH form, Eq. (8) and sum over all modes. By doing this we have taken all of the angular information of the transition multipole into account. Now to check this experimentally we need to measure an instance of the field, namely the photon. The presence of the absorber (the detector) collapses the wavefunction resulting in an absorption event. At this point all information regarding the angular distribution of the transition multipole is lost. If we want this we must make multiple measurements around the emitter and reconstruct the field profile. This has the physically appealing notion of requiring multiple photon modes to construct a field over all space. Consequently it is not possible to obtain information about the origin of the photon without multiple measurements.

Acknowledgments. We thank Prof. David Andrews for many insightful discussions, and for reading the manuscript. GAJ thanks Prof. Mark Lusk for thought provoking discussions.

\section{References}

1. E. Noether 1918, Math.- Phys. Kl. 2, 235 (1918)

2. D. E. Neuenschwander, Emmy Noether's Wonderful Theorem 2012 (Baltimore, MD: John Hopkins University Press)

3. D. L. Andrews, Phys. Rev. A, 81, 033825 (2010)

4. D. L. Andrews, Phys. Chem. Chem. Phys., 12, 7409 (2012)

5. V. B. Berestetskii, E. M. Lifshitz, and L. P. Pitaevskii, Quantum Electrodynamics, 2nd ed. (Butterworth, Oxford, UK, 1982), p. 18

6. A. S. Davydov and D. ter Haar, Quantum Mechanics, 1965, (Oxford, Pergamon Press), p 411

7. E. A. Power and T. Thirunamachandran, Phys. Rev. A, 28, 2649 (1983)

8. G. D. Scholes and D. L. Andrews, J. Chem. Phys. 107, 5374 (1997)

9. A. Salam, J. Chem. Phys. 122, 044112 (2005).

10. E. M. Rice, D. S. Bradshaw, K. Saadi and D. L. Andrews, Eur. J. Phys., 33, 345 (2012)

11. E. Nagali, F. Sciarrino, F. De Martini, L. Marrucci, B. Piccirillo, E. Karimi, E. Santamato, Phys. Rev. Lett., 103, 013601 (2009)

12. E. N. Bulgakov and A. F. Sadreev, Phys. Rev. A, 94, 033856 (2016)

13. F. Giammanco, A. Perona, P. Marsili, F. Conti, F. Fidecaro, S. Gozzini, A. Lucchesini, Opt. Lett., 42, 219 (2017)

14. B. S. Davis, L. Kaplan, J. H. McGuire, J. Opt. 15, 035403 (2013)

15. C. T. Schmiegelow, J. Schulz, H. Kaufmann, T. Ruster, U. G. Poschinger, F. Schmidt-Kaler, Nat. Comm. 7, 12998 (2016)

16. M. Babiker, C. R. Bennett, D. L. Andrews, L. C. Dávila Romero, Phys. Rev. Lett., 89, 143601 (2002)

17. L. Allen, M. J. Padgett and M. Babiker, Prog. Opt., 39, 291 (1999)

18. H. Rubinsztein-Dunlop, A. Forbes, M. V. Berry, M. R. Dennis, D. L. Andrews, M. Mansuripur, C. Denz, C. Alpmann, P. Banzer, T. Bauer, E. Karimi, L. Marrucci, M. Padgett, M. Ritsch-Marte, N. M. Litchinitser, N. P. Bigelow, C. Rosales-Guzmán, A. Belmonte, J. P. Torres, T. W. Neely, M. Baker, R. Gordon, A. B. Stilgoe, J. Romero, A. G. White, R. Fickler, A. E. Willner, G. Xie, B. McMorran, A. M. Weiner, J. Opt., 19, 013001 (2017)

19. R. D. Cowan, The Theory of Atomic Structure and Spectra, (University of California Press, Berkeley, 1981).

20. D. P. Craig, T. Thirunamachandran, Molecular Quantum Electrodynamics, (Dover, New York, 1998), chap. 10

21. A. Salam, Molecular Quantum Electrodynamics: Long-Range Intermolecular Interactions, (Wiley, New Jersey, 2010).

22. A. R. Edmonds, Angular Momentum in Quantum Mechanics (Princeton University Press, Princeton, NJ, 1957)

23. D. M. Brink and G. R. Satchler, Angular Momentum (Oxford University Press, Oxford, 1968).

24. R. Grinter, J. Phys. B: At., Mol. Opt. Phys. 41, 095001 (2008)

25. R. Grinter, J. Phys. B: At., Mol. Opt. Phys. 47, 075004 (2014).

26. R. Grinter, G. A. Jones, J. Chem. Phys., 145, 074107 (2016)

27. W. H. Zurek, Rev. Mod. Phys., 75, 715 (2003) 